\title{
Immunolocalization of Notch Signaling Protein Molecules in a Maxillary ChOndrosarcoma And Its RECURREnt Tumor
}

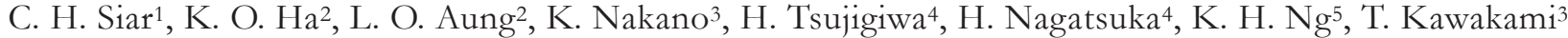 \\ ${ }^{1}$ Department of Oral Pathology, Oral Medicine \& Periodontology, Faculty of Dentistry, University of Malaya, Kuala Lumpur, Malaysia, \\ ${ }^{2}$ Department of Oral \& Maxillofacial Surgery, Faculty of Dentistry, University of Malaya, Kuala Lumpur, Malaysia, \\ ${ }^{3}$ Hard Tissue Pathology Unit, Matsumoto Dental University Graduate School of Oral Medicine, Shiojiri, Japan, \\ ${ }^{4}$ Department of Oral Pathology and Medicine, Okayama University Graduate School of Medicine, Dentistry and Pharmaceutical \\ Sciences, Okayama, Japan, \\ ${ }^{5}$ Formerly Unit of Stomatology, Cancer Research Centre, Institute for Medical Research, Jalan Pahang, Kuala Lumpur, Malaysia
}

\begin{abstract}
Background: Notch receptors are critical determinants of cell fate in a variety of organisms. Notch signaling is involved in the chondrogenic specification of neural crest cells. Aberrant Notch activity has been implicated in numerous human diseases including cancers; however its role in chondrogenic tumors has not been clarified.

Method: Tissue samples from a case of primary chondrosarcoma of the maxilla and its recurrent tumor were examined immunohistochemically for Notch1-4 and their ligands (Jagged1, Jagged2 and Delta1) expression.

Results: Both primary and recurrent tumors were histopathologically diagnosed as conventional hyaline chondrosarcoma (WHO Grade I). Hypercellular tumor areas strongly expressed Notch3 and Jagged1 in spindle and pleomorphic cells suggesting up-regulation of these protein molecules at sites of tumor proliferation. Expression patterns were distinct with some overlap. Differentiated malignant and atypical chondrocytes demonstrated variable expression levels of Jagged1, and weak to absent staining for Notch1, 4 and Delta1. Protein immunolocalization was largely membranous and cytoplasmic, sometimes outlining the lacunae of malignant chondrocytes. Hyaline cartilage demonstrated a diffuse or granular precipitation of Jagged1 suggesting presence of soluble Jagged1 activity at sites of abnormal chondrogenesis. No immunoreactivity for the other Notch members was observed. Calcified cartilage was consistently Notch-negative indicating down-regulation of Notch with cartilage maturation. Stromal components namely endothelial cells and fibroblasts variably expressed Notch1, 3 and Jagged1 but were mildly or non-reactive for the other members.

Conclusions: Results indicate that Notch signaling pathway may participate in cellular differentiation and proliferation in chondrosarcoma. Findings implicate Notch3 and Jagged1 as key molecules that influence the differentiation and maturation of cells of chondrogenic lineage.
\end{abstract}

Key words: Chondrosarcoma, Immunohistochemistry, Notch receptors, Notch ligands

\section{INTRODUCTION}

Chondrosarcoma is a rare malignant mesenchymal tumour characterized histopathologically by the production of cartilaginous tissue and the absence of production of bone tissue by the tumour cells [1-2]. It ranked as the second most common bone malignancy where it forms approximately $11 \%$ of all primary bone cancers [2-3]. Chondrosarcoma mostly affects long bones especially the pelvis, femur and humerus. It is uncommon in the head and neck region where its frequency of occurrence ranges from $1-12 \%$ of all chondrosarcomas [3-7]. Of these, $10 \%$ occur in the jawbones [6,7] and most of these arise in the maxilla with a predilection for the anterior maxillary region [3-4].

Chondrosarcomas are sub-classified into the conventional (hyaline/myxoid), dedifferentiated, clear cell, and mesenchymal subtypes [3-5]. Conventional chondrosarcoma, the most common subtype, is composed of either hyaline cartilage, myxoid cartilage or a combination of both of these matrices. The hyaline subtype is characterized by hypercellular hyaline cartilage containing cytologically atypical chondrocytes within lacunae. In contrast, the atypical chondrocytes of the myxoid subtype do not reside in lacunae but instead are enmeshed in a flocculent myxoid matrix. The mesenchymal subtype is known to display a more anaplastic appearance. According to the World Health Organization (WHO) grading system, three categories of chondrosarcoma were identified: grade I (well differentiated), grade II (moderately differentiated) and grade III (poorly differentiated) [1-2]. The biological behaviour of these tumors is characterized by progressive enlargement and subsequent compression or invasion of local structures such as cranial nerves. Complete surgical resection of these tumors is the most acceptable treatment of choice [7]. Radiotherapy and chemotherapy as adjunctive or palliative therapy remain controversial. 
The conserved Notch signaling pathway regulates cell proliferation, differentiation, and cell fate in diverse tissues [8-11]. Mammalian Notch family comprises four receptors (Notch1, Notch2, Notch3 and Notch4) and five ligands (Jagged1, Jagged2 Delta1, Delta2, Delta3 and Delta4). Notch receptors are structurally homologous transmembrane proteins with distinct differences in their extracellular and intracellular domains (ICD). Jagged (Serrate) and Delta proteins are also structurally related Tran membrane proteins with multiple epidermal growth factor (EGF)-like repeats and DSL motif (Delta, Serrate, Lag-2) in their extracellular domains [9-11]. Binding of Notch ligands to their receptors on neighboring cells induce proteolytic cleavages, releasing Notch ICD which translocates to the nucleus to interact with DNA-bound proteins. This in turn activates the transcription of selected target genes such as Hes1, Hes5, and Hes7 [8-11]. Dys-regulation of Notch signaling has been implicated in some genetic diseases and tumorigenesis [12-13]. Aberrant Notch can act as either a tumor promoter or a suppressor depending on the cell type and context. Regarding odontogenic neoplasms, we have reviewed the immunohistochemical characteristics of neoplasms and their physiological counterparts with Notch signaling [14]. In the manuscript, the data of published literatures suggested that Notch signaling plays some role in cytoplasmic differentiation or acquisition of tissue specific characteristics in neoplastic cells.

It has been demonstrated that markers for Notch signaling are differentially expressed during cartilage development and there is evidence for their functional role during this process where Notch1 is purportedly a marker for chondrogenic progenitor cells [15-16]. A search of the English language literature revealed that although aberrant Notch signaling has been detected in bone malignancies such as an osteosarcoma [17-18] and odontogenic jaw tumors [19-20], its activity in the chondrosarcoma has not been previously reported. The aim of this study was to investigate the potential role of Notch signaling during chondroid tumorigenesis by examining the immunolocalization pattern of Notch receptors and their ligands in a case of maxillary chondrosarcoma and its recurrent tumor.

\section{Materials AND Methods}

\section{Tissue SAMPLES}

Formalin-fixed, paraffin-embedded tissue blocks of a case of primary chondrosarcoma and its recurrence were retrieved and new $5 \mu \mathrm{m}$ thick sections were prepared for staining with hematoxylin-eosin, and for immunohistochemistry.

\section{IMMUNOHISTOCHEMISTRY}

Immunohistochemistry was performed against Notch1, Notch2, Notch3, Notch4, Jagged1, Jagged2 and Delta1 according to the Envision technique as previously described [19]. Briefly, deparaffinized sections of $5 \mu \mathrm{m}$ thickness were pretreated for antigen retrieval by microwaving $\left(99^{\circ} \mathrm{C}\right)$ in $10 \mathrm{mM}$ of citrate buffer $(\mathrm{pH} 6$, $20 \mathrm{~min})$. These sections were then immersed in $0.3 \%$ methanol containing 3\% hydrogen peroxide for 20 min, to block endogenous peroxidase, and rinsed in $0.05 \mathrm{M}$ Tris-buffered saline (TBS) (5 min, two times) before immersing in blocking solution (Dako Corporation, CA, USA) for $20 \mathrm{~min}$ at room temperature. Then the sections were incubated with the primary antibody against each of the Notch family members [mouse anti-human Notch1 monoclonal antibody (LSC16925), rabbit anti-human Notch2 polyclonal antibody (LS-B399), rabbit anti-human Notch3 polyclonal antibody (LS-B3399), rabbit anti-human Notch4 polyclonal antibody (LS-C40785), LifeSpan Biosciences Inc, Seattle, WA, USA, 1:500 dilution] and ligands [rabbit anti-human Jagged1 polyclonal antibody (LSB2442) and rabbit anti-mouse Delta1 polyclonal antibody (LS-B72), LifeSpan Biosciences Inc, Seattle, WA, USA, 1:500 dilution], [rabbit anti-human Jagged2 polyclonal antibody (ab60041), Abcam Inc, Cambridge, MA, USA, 1:500 dilution], for $1 \mathrm{~h}$ at room temperature. Immunoreactions were performed using the Envision Kit (Dako Corporation, CA, USA). The antigenic sites were visualized using diaminobenzidine (DAB) substrate chromogen (Dako Corporation, CA, USA) and counterstained with Mayer's hematoxylin. For negative control, sections were treated as above but without the primary antibody. All the control sections were negative. Appropriate positive controls were also applied.

\section{RESULTS}

Histological Findings

(Fig. 1A)

Microscopic examination of the primary and recurrent chondrosarcomas revealed lesional tissues composed of hypercellular hyaline cartilage containing cytologically atypical chondrocytes within lacunae (Fig. 1A). The histological diagnosis made was conventional or Grade I chondrosarcoma for both the primary and recurrent tumor.

\section{IMMUNOHISTOCHEMICAL FINDINGS}

$$
\text { (Fig. 1B-D, 2A-D) }
$$

In the hypercellular tumor areas, spindle-shaped and pleomorphic cells strongly expressed Notch3 (Fig. 1D) and Jagged1 (Fig. 2B) but were weakly positive for Notch1, Notch4 (Fig.2A) and weak to non-reactive for Delta1 (Fig. 2D). Notch2 (Fig. 1C) and Jagged2 (Fig. 2C) were not detected.

In the hyaline cartilage areas, the malignant and atypical chondrocytes stained heterogeneously for Jagged1 (Fig. 2B), and to a lesser extent Notch1 (Fig. 1B), Notch4 (Fig. 2A) and Delta1 (Fig. 2D). Protein immunolocalization was largely membranous and cytoplasmic, sometimes outlining the lacunae of chondrocytes. In addition Jagged1 was also detected as a diffuse or dotted positivity in the chondroid matrix. Occasional concentric spiral pattern of protein deposition was also observed (Fig. 2B). No immunoreactivity for the other Notch members was observed. Calcified cartilage was consistently negative for both Notch receptors and ligands. Stromal components namely blood vessels and fibroblasts stained variously for 

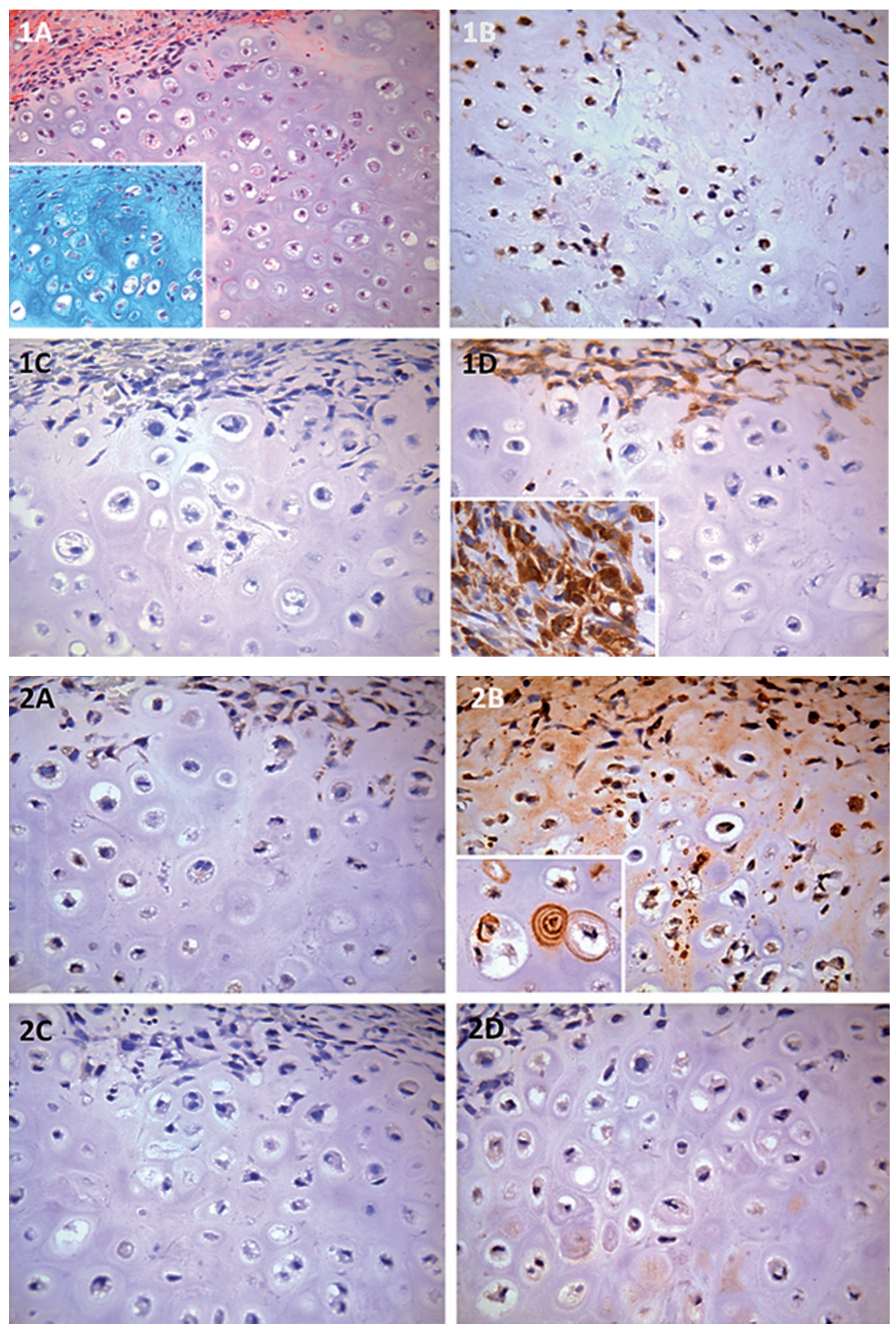
Fig. 1. Photomicrographs of chondrosarcoma showing: A and Inset, lesional areas composed of hyaline cartilage with hypercellular zone at the periphery; B, Notch1 moderate expression in atypical chondrocytes; C, Notch2 was not detected; D and Inset, Notch3 strong expression in spindle and pleomorphic cells but absence of activity in atypical chondrocytes (original magnification, A-D, x400; A, HE staining, A Inset, Alcian blue; B, anti-Notch1; C, anti-Notch2; D and Inset, anti-Notch3).

Fig. 2. Photomicrographs of chondrosarcoma showing: A, Notch4 weak expression in pleomorphic cells and atypical chondrocytes; B, and Inset, Jagged1 strong expression in pleomorphic cells, moderate to weak in atypical chondrocytes; diffuse positivity of chondroid matrix. Note concentric spiral protein deposition; C, Jagged2 was not detected; and D, Delta1 weak to absent expression in spindle and pleomorphic cells and atypical chondrocytes (original magnification, A-D, x400; A, anti-Notch4; B and Inset, anti-Jagged1; C, anti-Jagged2; D, anti-Delta1).

Notch1, 3, Jagged1 but were non-reactive for Notch2, Jagged 2 and Delta1.

\section{DiscusSION}

The primary chondrosarcoma tumor sample examined here was from a 58-year-old Malay female who had nasopharyngeal carcinoma treated by chemoradiotherapy about 10 years previously. The recurrent tumor that developed seven months later was also from the same patient. Both primary and recurrent tumors were histologically diagnosed as conventional chondrosarcoma (WHO Grade I). Although most chondrosarcomas arise de novo, they are common in patients with Ollier's disease, Maffucci syndrome, Paget's disease and osteochondroma [1-2]. It is likely that the chondrosarcoma that developed in this patient is an outcome complication of her treatment.

Positive immunolocalization of Notch receptor and ligand protein in the primary and recurrent chondrosarcomas investigated in this study suggests that Notch canonical pathway was activated during the development of this neoplasm. The expression patterns of the various Notch members were distinct with some overlap. Strong expression for Notch3 and Jagged 1 in the spindle and pleomorphic cells located in the hypercellular areas of the tumor suggests that these are key molecules that influenced the cell stemness of the chondrogenic progenitor cells. Reduced expression levels of Jagged1, Notch1, Notch4 and Delta1 in atypical chondrocytes implies that chondrocyte differentiation is associated with downregulation of these molecules. It has been shown that Jagged1mediated Notch signaling in human bone marrow stem cells is necessary to initiate chondrogenesis but must be switched off for chondrogenesis to proceed [15]. Similarly, in this study, we observed that Jagged1 was detected in the neoplastic chondroid matrix confirming that this molecule is also important as a mediator in Notch signaling during oncogenic chondrogenesis. In addition, as with others [21], this study too demonstrates that soluble Notch ligands can interact with membrane-bound Notch receptors to initiate proteolysis thus dispelling earlier views that Notch signaling necessitates interaction between membranebound Notch receptors and with membrane-bound ligands of adjacent cells.

In summary, a case of primary chondrosarcoma of the maxilla and its subsequent recurrent tumor were examined for Notch signaling activity. The data from this case study indicated that this pathway most likely plays an active and differential role in the acquisition of tissue-specific characteristics in ythis malignant car- tilaginous neoplasm. Current findings implicated Notch3 and Jagged1 as key molecules that influence cell fate decisions in tumoral cells of a chondrogenic lineage.

Acknowledgements: This study was partially funded by the University of Malaya Research Grant (RG083/09HTM) and Grant-in-aid for Scientific Research (C) (20592349) from the Japan Society for the Promotion of Science.

\section{REFERENCES}

1. Fletcher CDM, Unni K, Mertens K. Pathology and genetics of tumours of soft tissue and bone. World Health Organization, Geneva, 2002

2. Koch BB, Karnell LH, Hoffman HT, Apostolakis LW, Robinson RA, Zhen W, Menck HR. National cancer database report on chondrosarcoma of the head and neck. Head Neck 2000; 22: 408-425.

3. Prado FO, Nishimoto IN, da Cruz Perez DE, Kowalski LP, Lopes MA. Head and neck chondrosarcoma: Analysis of 16 cases. Br J Oral Maxillofac Surg 2009; 47: 555-557.

4. Mohammadinezhad C. Chondrosarcoma of the jaw. J Craniofac Surg 2009; 20: 2097-2100.

5. van Damme PA, de Wilde PC, Koot RA, Bruaset I, Slootweg PJ, Ruiter DJ. Juxtacortical chondrosarcoma of the mandible: report of a unique case and review of the literature. Int J Oral Maxillofac Surg 2005; 34: 94-98.

6. Saini R, Abd Razak NO, Ab Rahman S, et al. Chondrosarcoma of the mandible: a case report. J Can Dent Assoc 2007; 73:175-178.

7. Shirato T, Onizava K, Yamagata K, Yusa H, Iljima T, Yoshida H. Chondrosarcoma of the mandibular symphysis. Oral Oncol 2006; 43: 247-250.

8. Miele L. Notch signaling. Clin Cancer Res 2006; 12:10741079.

9. Fiúza UM, Arias AM. Cell and molecular biology of Notch. J Endocrinol 2007; 194: 459-474.

10. Fortini ME. Notch Signaling: The core pathway and its posttranslational regulation. Dev Cell 2009; 16: 633-647.

11. Artavanis-Tsakonas S, Rand MD, Lake RJ. Notch signaling: cell fate control and signal integration in development. Science 1999; 284: 770-776.

12. Harper JA, Yuan JS, Tan JB, Visan I, Guidos CJ. Notch signaling in development and disease. Clin Genet 2003; 64: 461-472.

13. Leong KG, Karsan A. Recent insights into the role of Notch signaling in tumorigenesis. Blood 2006; 107: 22232233.

14. Nakano K, Nagatsuka H, Tsujigiwa H, Gunduz M, Katase N, Siar CH, Kawakami T. Immunohistochemical characteristics of odontogenic neoplasms and their physiological counterparts. J Hard Tissue Biol 2008: 79-90.

15. Ijuin k, Nakanishi K, Ito K. Different downstream pathways for Notch signaling are required for gliogenic and chondrogenic specification of mouse mesencephalic neural crest cells. Mech Dev 2008; 125: 462-474. 
16. Oldershaw RA, Hardingham TE. Notch signaling during chondrogenesis of human bone marrow stem cells. Bone 2010; 46: 286-293.

17. Kawakami T, Siar CH, Ng KH, Shimizu T, Okafuji N, Kurihara S, Tsujigiwa H, Nagatsuka H, Nagai N. Expression of Notch in a case of osteosarcoma of the maxilla. Eur J Med Res 2004; 9: 1-3.

18. Kawakami T, Shimizu T, Kimura A, Hasegawa H, Siar $\mathrm{CH}, \mathrm{Ng} \mathrm{KH}$, Nagatsuka H, Nagai N, Kanda H. Immunohistochemical examination of cytological differentiation in osteosarcomas. Eur J Med Res 2005; 10: 475-479.

19. Siar CH, Nakano K, Ng KH, Tomida M, Nagatsuka H, Kawakami T. Squamous odontogenic tumor of the mandible: A case report demonstrating immunoexpression of Notch1, 3, 4, Jagged1 and Delta1. Eur J Med Res 2010; 15: 180-184

20. Nakano K, Siar CH, Tsujigiwa H, Nagatsuka H, Nagai N, Kawakami T. Notch signaling in benign and malignant ameloblastic neoplasms. Eur J Med Res 2008; 13: 476480
21. Masuya M, Katayama N, Hoshino N, Nishikawa H, Sakano S, Araki H, Mitani H, Suzuki H, Miyashita H, Kobayashi K, Nishii K, Minami N, Shiku H. The soluble Notch ligand, Jagged-1, inhibits proliferation of CD34+ macrophage progenitors. Int J Hematol 2002; 75: 269276.

Received: May 24, 2010 / Accepted: July 21, 2010

Address for correspondence:

Toshiyuki Kawakami, PhD, Professor

Hard Tissue Pathology Unit

Matsumoto Dental University Graduate School

of Oral Medicine

1780 Hirooka-Gobara

Shiojiri, 399-0781

Japan

Phone and Fax: +81-263-51-2035

E-mail: $\quad$ kawakami@po.mdu.ac.jp 